\title{
Effect of NC-1100 [1-(3,4-Dimethoxyphenyl)-2- (4-Diphenylmethylpiperazinyl) Ethanol Dihydrochloride] on $r$-Aminobutyric Acid (GABA) Metabolism in Rat Brain: Analysis Using Stroke-Prone Spontaneously Hypertensive Rat
}

\author{
Tsuneichi HASHIMOTO, Misa KIMORI, Yoshiko NAKAMURA \\ and Kinya KURIYAMA
}

Department of Pharmacology, Kyoto Prefectural University of Medicine. Kamikyo-ku, Kyoto 602, Japan

Accepted February 25, 1989

\begin{abstract}
Effects of oral administration of NC-1100 on the metabolism of neuroactive amino acids in rat brain were studied using stroke-prone spontaneously hypertensive (SHR-SP) and Wistar Kyoto rats. The repeated administration of $\mathrm{NC}-1100$ induced a significant increase of $\gamma$-aminobutyric acid (GABA) content in the cerebellum and medulla oblongata of SHR-SP. The decrease of aspartic acid contents in the cerebellum and medulla oblongata of SHR-SP was also noted following NC-1100 administration. Although the activity of L-glutamic acid decarboxylase did not change in these cerebral areas, the activity of GABAtransaminase:succinic semialdehyde dehydrogenase was found to be significantly reduced in the cerebellum of SHR-SP following the repeated administration of NC-1100. The turnover rate of GABA was also significantly reduced in the cerebellum and medulla oblongata of SHR-SP. It was also found that the spontaneous release of preloaded $\left[{ }^{3} \mathrm{H}\right] \mathrm{GABA}$ from cerebral cortical slices was significantly retarded by the continuous oral administration of NC-1100. These results suggest that NC-1100 may be a drug inducing the increase of GABA in the cerebellum and medulla oblongata following continuous administration, especially in animals having hypertension associated cerebrovascular disorders such as SHRSP.
\end{abstract}

NC-1100 [1-(3,4-dimethoxyphenyl)-2-(4diphenylmethylpiperazinyl) ethanol dihydrochloride] has been developed as a drug having a selective effect on cerebral blood flow and possible therapeutic efficacy on various neurological disorders associated with cerebral hemorrhage and apoplexy. Although various antithrombotic and vasodilating drugs have been used for the treatment of cerebrovascular diseases, most of these drugs have no selective effect on target organs as well as blood vessels. As a result of this nonselective effect, general vasodilators often induce the reduction of blood flow in the brain (1) as the result of peripheral vasodilation.
Taurine (2-aminoethanesulfonic acid) and glycine are suspected to be a neurotransmitter and/or a neuromodulator in mammalian brain (2), while $r$-aminobutyric acid (GABA) is considered to be a potential candidate for an inhibitory neurotransmitter in the mammalian central nervous system (CNS). On the other hand, glutamic and aspartic acids are proposed to be excitatory neurotransmitters in the CNS.

In this study, we have investigated the effect of continuous administration of $\mathrm{NC}$ 1100 on the content and metabolism of these neuroactive amino acids using normotensive Wistar Kyoto (WKY) and stroke-prone spontaneously hypertensive (SHR-SP) rats. 
respectively.

\section{Materials and Methods}

Chemicals: GABA, glutamic acid, $\beta$ aminoethylisothiouronium bromide hydrochloride (AET), pyridoxyl-5'-phosphate (PLP) and aminooxyacetic acid (AOAA) were purchased from Sigma Chemical $C o$. (St. Louis, MO). DL-[1-14 C]-glutamic acid (47 $\mathrm{mCi} / \mathrm{mmol}), \quad \gamma-\left[2,3-{ }^{3} \mathrm{H}\right]$-aminobutyric acid $(70 \mathrm{Ci} / \mathrm{mmol})$ and $\left[\right.$ methylene- $\left.{ }^{3} \mathrm{H}(\mathrm{N})\right]$ muscimol $(30.8 \mathrm{Ci} / \mathrm{mmol})$ were obtained from New England Nuclear (Boston, MA). All other chemicals used were of commercially guaranteed grade.

Animal handling and preparation of tissue sample: Male SHR-SP (F-15-F-16) and WKY rats, 15-18 weeks of age, were used throughout this study. Animals received orally $100 \mathrm{mg} / \mathrm{kg}$ of NC-1100 once a day for 7 days. One hour after the last administration, rats were killed by focussed microwave irradiation $(5 \mathrm{~kW}, 0.8 \mathrm{sec})$ to determine the content of amino acids or by decapitation to examine the activity of various enzymes. The brain was dissected out on a chilled plastic plate into seven regions: cerebral cortex, cerebellum, midbrain, medulla oblongata, striatum, hypothalamus and hippocampus. as described by Glowinski and Iversen (3).

Determination of neuroactive amino acids: Each brain region from animals killed by focussed microwave irradiation was dissected and homogenized in 50 volumes of $5 \%$ trichloroacetic acid, and the homogenate was centrifuged at $8000 \times \mathrm{g}$ for $30 \mathrm{~min}$ at $4^{\circ} \mathrm{C}$. The supernatant obtained was then used for the assay of various neuroactive amino acids. For the measurement of amino acids, a high performance liquid chromatography (HPLC) system, Model LC-3A (Shimadzu Seisakusho, Ltd., Kyoto, Japan), was used. The HPLC system employed was essentially the same as previously reported by Ida and Kuriyama (4). Briefly, the column $(150 \times 4 \mathrm{~mm}$ i.d.) packed with cation-exchange resin. ISC-07/S1504, was used at $55^{\circ} \mathrm{C}$. Three types of eluent $[0.067 \mathrm{~N}$ sodium citrate $(\mathrm{pH} 3.25), 0.067 \mathrm{~N}$ sodium citrate $(\mathrm{pH} 4.25)$ and $0.2 \mathrm{~N}$ sodium citrate $(\mathrm{pH} 9.00)]$ were used with the flow rate of $0.5 \mathrm{ml} / \mathrm{min}$. Amino acids were detected using o-phthalaldehyde, and the fluorescence of each eluent was measured spectrofluorometrically at excitation and emission wavelengths of 348 and $450 \mathrm{~nm}$, respectively.

Assay of enzyme activities: To determine the activity of $\mathrm{L}$-glutamic acid decarboxylase (GAD L-glutamate-decarboxylase, EC4.1.1.15). which catalyzes the formation of GABA from L-glutamic acid (Glu) and plays an important role as the rate limiting enzyme in GABA biosynthesis, each brain region obtained from animals killed by decapitation was homogenized in 10 volumes of $\mathrm{N}_{2}$ gassed deionized water, and the homogenate was incubated with $0.033 \mu \mathrm{Ci}$ of D,L-[1$\left.{ }^{14} \mathrm{C}\right] \mathrm{Glu}$ in a mixture containing $140 \mathrm{mM}$ potassium phosphate buffer $(\mathrm{pH} 6.4), 1.4$ $\mathrm{mM} \mathrm{AET}$, and $50 \mathrm{mM}$ carrier Glu in a microvessel, specially designed by Kimura and Kuriyama (5), at $37^{\circ} \mathrm{C}$ for $90 \mathrm{~min}$ in the presence of PLP. The activity of 4 -aminobutyrate:2-oxoglutarate aminotransferase (GABA-T. EC2.6.1.19), a GABA degrading enzyme, was determined by measuring the fluoresence of NADH formed from NAD which was coupled with the enzymatic reaction. Each cerebral tissue was homogenized in 50 volumes of $\mathrm{N}_{2}$-gassed deionized water containing $0.4 \mathrm{mM}$ PLP and $0.1 \%$ Triton $X-100$, and the homogenate was incubated with $60 \mathrm{mM}$ pyrophosphate buffer $(\mathrm{pH}$ 8.6), $20 \mathrm{mM}$ GABA, $5 \mathrm{mM} \alpha$-ketoglutarate, $3 \mathrm{mM}$ NAD and $0.005 \%$ mercaptoethanol at $37^{\circ} \mathrm{C}$ for $90 \mathrm{~min}$. After the termination of reaction by adding $0.08 \mathrm{~N}$ sodium hydroxide, $10 \mathrm{~N}$ sodium hydroxide containing $0.03 \%$ hydrogen peroxide was added to the mixture to obtain the maximum fluorescence, and it was then incubated at $60^{\circ} \mathrm{C}$ for $15 \mathrm{~min}$. The fluorescence of each tube was measured spectrofluorometrically at emission and excitation wavelengths of 370 and $460 \mathrm{~nm}$. respectively.

Measurement of turnover of GABA in vivo: The metabolic turnover of GABA in the brain was assayed by the methods of Löscher (6). Rats that had received intraperitoneally 30 $\mathrm{mg} / \mathrm{kg}$ of AOAA, an inhibitor of GABAtransaminase, in addition to the oral administration of NC-1100, were sacrificed by microwave irradiation at $60 \mathrm{~min}$ after the administration and subjected to the measurement of cerebral GABA content. The turnover 
rate in vivo was then estimated from the difference of cerebral GABA contents in AOAA-injected and noninjected animals.

Determination of $\left[{ }^{3} \mathrm{H}\right] \mathrm{GABA}$ uptake in cerebral slices: Following decapitation, the cerebral cortex, cerebellum and medulla oblongata were dissected out from each brain on a chilled plastic plate. The slices of each cerebral region were then prepared using a microslicer in cold Krebs-Ringer Tris- $\mathrm{HCl}$ (KRT) buffer ( $\mathrm{pH} 7.4$ ) which was equilibrated with an atmosphere of $95 \% \mathrm{O}_{2}$ and $5 \% \mathrm{CO}_{2}$. Each slice (200 $\mu \mathrm{m}$ in thickness) was preincubated for $15 \mathrm{~min}$ at $25^{\circ} \mathrm{C}$ in $10 \mathrm{ml}$ of $\mathrm{KRT}$ to reduce endogeneous GABA. After the preincubation, the cerebral slices were incubated for $20 \mathrm{~min}$ at $25^{\circ} \mathrm{C}$ with $2 \mathrm{ml}$ of the KRT containing $2 \mu \mathrm{M}\left[{ }^{3} \mathrm{H}\right] \mathrm{GABA}$ and $1 \times 10^{-4}$ $M$ AOAA to measure the uptake. The reaction was terminated by rapidly rinsing the slices three times with $5 \mathrm{ml}$ of ice-cold KRT buffer. The washed slices were then placed into a scintillation vial containing $0.3 \mathrm{ml}$ of methylbenzetonium hydroxide and kept for several hours to dissolve the tissues. The radioactivity was counted on a Packard 3255 liquid scintillation spectrometer.

Determination of $\left[{ }^{3} \mathrm{H}\right] \mathrm{GABA}$ release: After dissecting the brain, each brain tissue was cut into slices (200 $\mu \mathrm{m}$ in thickness) using a microslicer, and the slices were preincubated in $10 \mathrm{ml}$ of KRT buffer at $25^{\circ} \mathrm{C}$ for 20 min in a small plastic container equipped with a nylon mesh at the bottom. The container was then transferred to a vial containing $1 \mathrm{ml}$ of $\mathrm{KRT}$ medium and $2 \mu \mathrm{M}\left[{ }^{3} \mathrm{H}\right] \mathrm{GABA}$ and incubated at $25^{\circ} \mathrm{C}$ for $30 \mathrm{~min}$ to preload the slices with $\left[{ }^{3} \mathrm{H}\right] \mathrm{GABA}$. For the measurements of release. a small plastic container with slices was successively transferred to vials containing $1 \mathrm{ml}$ of KRT buffer in the presence of AOAA $\left(5 \times 10^{-4} \mathrm{M}\right)$ at $25^{\circ} \mathrm{C}$ with an interval of $2 \mathrm{~min}$, and these transfer procedures were continued up to $30 \mathrm{~min}$. High potassium ( $30 \mathrm{mM}$ ) stimulation was applied at $16 \mathrm{~min}$ after the initiation of incubation by removing an equimolar concentration of sodium chloride. The spotaneous release was also measured using a normal concentration of $\mathrm{KCl}$ in the medium. The radioactivity in each vial was counted using a liquid scintillation spectrometer after adding $20 \mathrm{ml}$ of the Triton-toluene scintillator.
At the end of the release measurement, radioactivity remaining in the tissue was also determined after solubilizing with methylbenzetonium hydroxide. The release of $\left[{ }^{3} \mathrm{H}\right]$ GABA was expressed as a percentage of the total radioactivities accumulated (sum of the radioactivity released and the radioactivity remaining in the tissue at the end of the incubation). Kinetic parameters such as the fraction rate constant $(K)$, the time required for decrease to half value of the component $\left(t_{1 / 2}\right)$ and percent increase were calculated as described previously (7).

Assay of $\left[{ }^{3} \mathrm{H}\right]$ muscimol binding: The specific binding of $\left[{ }^{3} \mathrm{H}\right]$ muscimol to the $\mathrm{GABA}_{A}$ receptor was determined as described by Ito and Kuriyama (8). In brief, $0.5 \mathrm{ml}$ of synaptic membrane suspension obtained from the cerebellum, medulla oblongata and other brain areas was incubated for $30 \mathrm{~min}$ at $2^{\circ} \mathrm{C}$ with $10 \mathrm{nM}$ of $\left[{ }^{3} \mathrm{H}\right]$ muscimol in $50 \mathrm{mM}$ Tris-citrate buffer. Following the incubation. the mixture was filtered under vacuum through a Whatman GF/B glass-fiber filter. The filter was then washed 3 times with $3 \mathrm{ml}$ of $50 \mathrm{mM}$ Tris-citrate buffer containing $8 \%$ polyethyleneglycol at $2{ }^{\circ} \mathrm{C}$. The specific binding of $\left[{ }^{3} \mathrm{H}\right]$ muscimol was defined as the total binding of $\left[{ }^{3} \mathrm{H}\right]$ muscimol minus the binding obtained in the presence of $10^{-3} \mathrm{M}$ GABA.

Determination of protein content: Protein content was determined by the method of Lowry et al. (9) using bovine serum albumin as standard.

Analysis of data: Statistical significance of data was analyzed by Student's $t$-test, and data are presented as the mean \pm S.E.M.

\section{Results}

Regional disribution of GABA content in the brains of WKY and SHR-SP: As shown in Table 1, it was found that GABA content in the cerebellum, hypothalamus and hippocampus of SHR-SP showed a significant decrease as compared with those of WKY.

Effect of continuous administration of NC1100 on neuroactive amino acid contents in the brain: Continuous oral administration of NC-1100 (100 mg/kg. p.0., 7 days) did not induce any significant changes in amino acid contents in the brain of WKY, except for 
Table 1. Regional distribution of $\gamma$-aminobutyric acid (GABA) content in the brains of WKY and SHR-SP

\begin{tabular}{lcc}
\hline & \multicolumn{2}{c}{ GABA content $(\mu \mathrm{mol} / \mathrm{g}$ wet weight) } \\
\cline { 2 - 3 } & WKY & SHR \\
\hline Cerebral cortex & $1.82 \pm 0.19$ & $1.25 \pm 0.20$ \\
Cerebellum & $2.58 \pm 0.28$ & $1.11 \pm 0.05^{*}$ \\
Midbrain & $2.73 \pm 0.46$ & $2.24 \pm 0.21$ \\
Medulla oblongata & $1.78 \pm 0.52$ & $1.12 \pm 0.17$ \\
Striatum & $3.88 \pm 1.35$ & $2.81 \pm 0.50$ \\
Hypothalamus & $5.52 \pm 0.38$ & $2.26 \pm 0.06^{* *}$ \\
Hippocampus & $2.97 \pm 0.26$ & $1.39 \pm 0.14^{* *}$ \\
\hline
\end{tabular}

Each value represents the mean \pm S.E.M. obtained from 3 to 4 separate experiments. ${ }^{*} P<0.01,{ }^{*} P<0.001$ compared with each WKY value.

Table 2. Effect of continuous oral administration of NC-1100 on $\left[{ }^{3} \mathrm{H}\right] \mathrm{GABA}$ release from cerebral slices of SHR-SP

\begin{tabular}{|c|c|c|c|c|c|c|}
\hline & & \multicolumn{2}{|c|}{ Rapid phase } & \multicolumn{2}{|c|}{ Slow phase } & \multirow{2}{*}{$\begin{array}{l}\text { High } \mathrm{K}^{+} \text {evoked } \\
\text { release } \\
\text { (percent increase) }\end{array}$} \\
\hline & & $K\left(\min ^{-1}\right)$ & $\mathrm{t}_{1 / 2}(\min )$ & $K\left(\min ^{-1}\right)$ & $\mathrm{t}_{1 / 2}(\min )$ & \\
\hline \multirow[t]{2}{*}{ Cerebral cortex } & Control & $1.11 \pm 0.27$ & $0.70 \pm 0.12$ & $0.0159 \pm 0.0031$ & $53.2 \pm 17.1$ & $645.6 \pm 149.6$ \\
\hline & $N C-1100$ & $1.03 \pm 0.08$ & $0.68 \pm 0.06$ & $0.0139 \pm 0.0025$ & $56.0 \pm 11.9$ & $609.5 \pm 67.4$ \\
\hline \multirow[t]{2}{*}{ Cerebellum } & Control & $1.08 \pm 0.20$ & $0.71 \pm 0.14$ & $0.0176 \pm 0.0043$ & $46.5 \pm 10.3$ & $188.0 \pm 42.0$ \\
\hline & NC- 1100 & $1.07 \pm 0.15$ & $0.69 \pm 0.11$ & $0.0085 \pm 0.0006$ & $83.0 \pm 6.5^{*}$ & $168.0 \pm 9.8$ \\
\hline \multirow[t]{2}{*}{ Medulla oblongata } & Control & $0.72 \pm 0.08$ & $0.84 \pm 0.15$ & $0.0197 \pm 0.0056$ & $43.4 \pm 10.5$ & $134.5 \pm 16.5$ \\
\hline & $N C-1100$ & $0.88 \pm 0.15$ & $0.85 \pm 0.13$ & $0.0065 \pm 0.0009$ & $114.6 \pm 21.2^{*}$ & $149.3 \pm 17.3$ \\
\hline
\end{tabular}

NC-1100 (100 mg/kg) was given orally once daily for 7 days. $\quad P<0.05$, compared with each control value. The data represents the mean $\pm S$. E.M. of 4 to 5 separate experiments. 
taurine content in the cerebellum (Fig. 1). On the other hand, the continuous oral administration of $\mathrm{NC}-1100(100 \mathrm{mg} / \mathrm{kg}$. p.o., 7 days) induced a significant increase of GABA content in the cerebellum and medulla oblongata of SHR-SP (Fig. 2). In the SHR-
SP, continuous administration of NC-1100 also resulted in a significant decrease of aspartic acid content in the cerebellum and medulla oblongata. In addition, it was found that cerebellar taurine content in WKY and SHR-SP decreased following oral adminis-

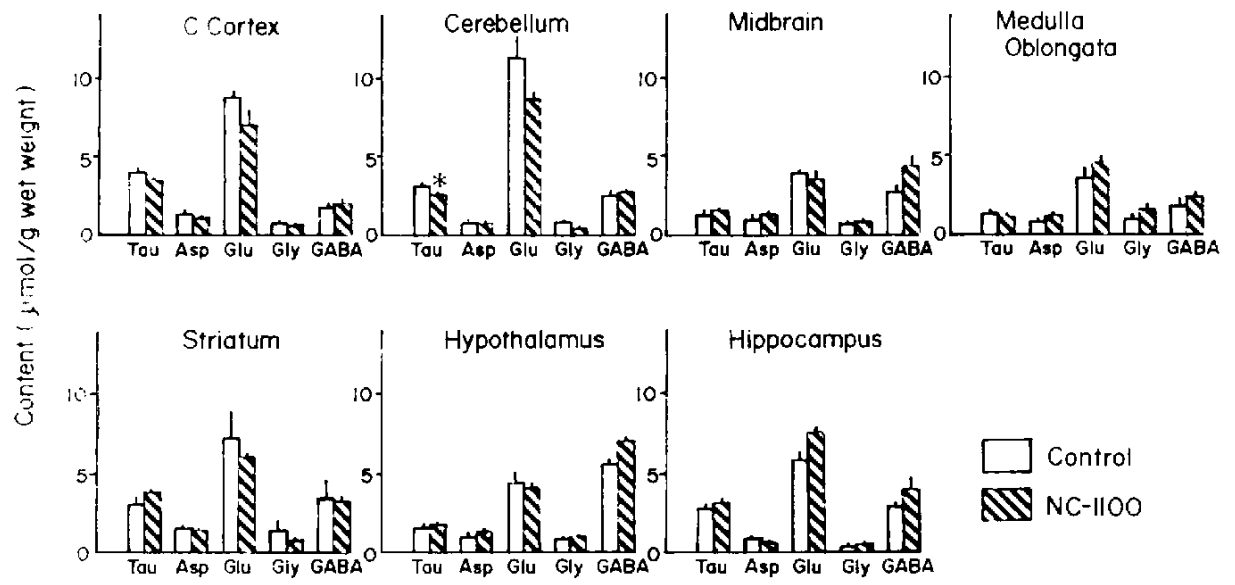

Fig. 1. Effect of continuous oral administration of NC-1100 on neuroactive amino acid contents in the brain of Wistar Kyoto rat. Rats were given orally $100 \mathrm{mg} / \mathrm{kg}$ of NC-1100 once a day for 7 days. Each value represents the mean \pm S.E.M. obtained from 4 separate experiments. ${ }^{*} P<0.05$, compared with each control value. Tau: taurine; ASP: aspartic acid; Glu:glutamic acid; Gly: glycine; GABA: $\gamma$-aminobutyric acid.

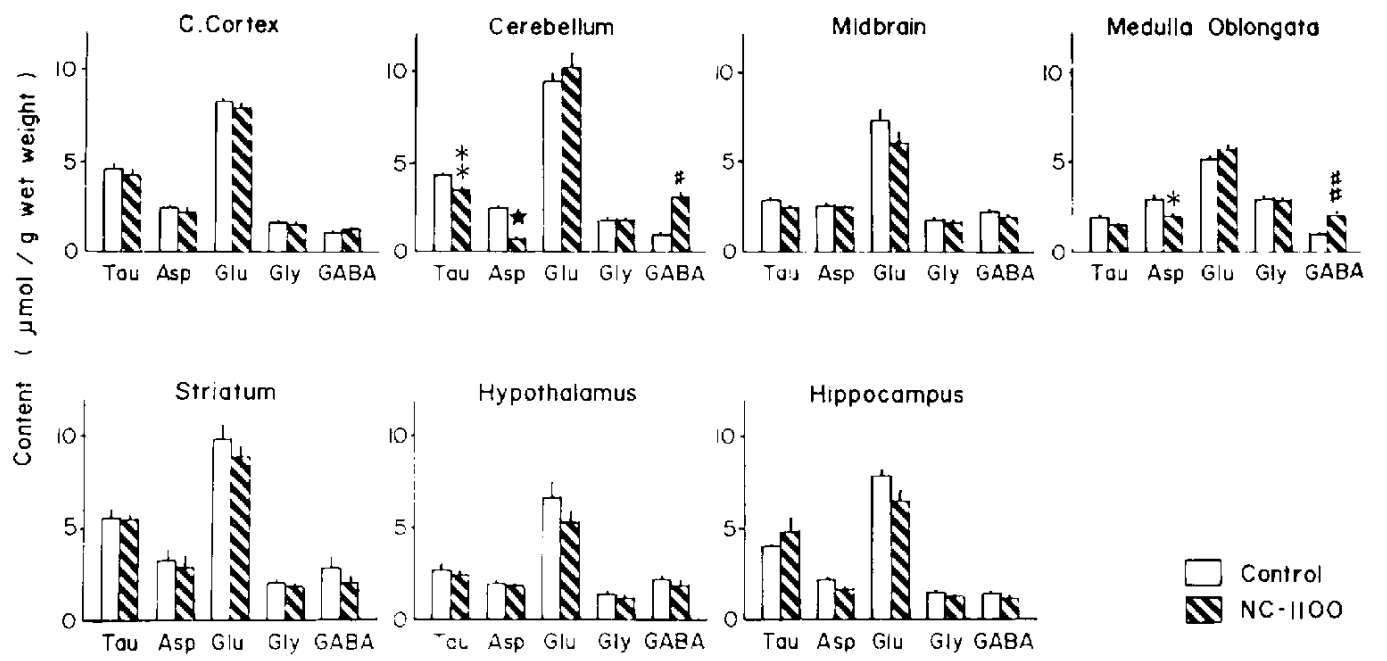

Fig. 2. Effect of continuous oral administration of NC-1100 on neuroactive amino acid contents in the brain of SHR-SP. NC-1100 $(100 \mathrm{mg} / \mathrm{kg})$ was given orally once a day for 7 days. Each value indicates the mean \pm S.E.M. obtained from 4 separate experiments. ${ }^{*} P<0.05,{ }^{*} P<0.02, \# P<0.01, \# P<0.005$ and $\star P<0.001$, compared with each control value. Abbreviations are the same as in Fig. 1. 
tration of $\mathrm{NC}-1100$. The contents of other neuroactive amino acids such as glutamic acid and glycine were not affected in any brain areas of WKY and SHR-SP following the continuous administration of NC-1100 (Figs. 1 and 2).

Effect of continuous oral administration of NC-1100 on GAD and GABA-T activities in the brain: To elucidate biochemical mechanisms underlying the increase of GABA induced by the continuous administration of NC-1100 in the specific regions of rat brain, the activities of GAD and GABA-T, which are responsible for the formation and degradation of GABA, respectively, were examined. Although these enzyme activities were not affected by the administration of NC-1100 to WKY. GABA-T activity in the cerebellum of SHR-SP showed a significant decrease following the repeated administration of NC-1100 (Fig. 3), which coincided with the increase of GABA in this brain area (Fig. 2). No significant change, however, was observed in GAD activities in any brain areas of SHR-SP (Fig. 3) as well as those of WKY.

Effect of oral administration of NC-1100 on turnover rate of GABA in vivo in the brains of WKY and SHR-SP: To estimate the cerebral turnover of GABA in vivo, a low dose of AOAA $(30 \mathrm{mg} / \mathrm{kg}$, i.p.) was injected to NC1100 treated and non-treated animals. The AOAA administration caused a significant increase of GABA levels in the brain linearly up to $3 \mathrm{hr}$. Continuous oral administration of NC1100 to SHR-SP resulted in a significant decrease in the turnover rate of GABA in the cerebellum and medulla oblongata (Fig. 4). No significant alteration in the turnover rate

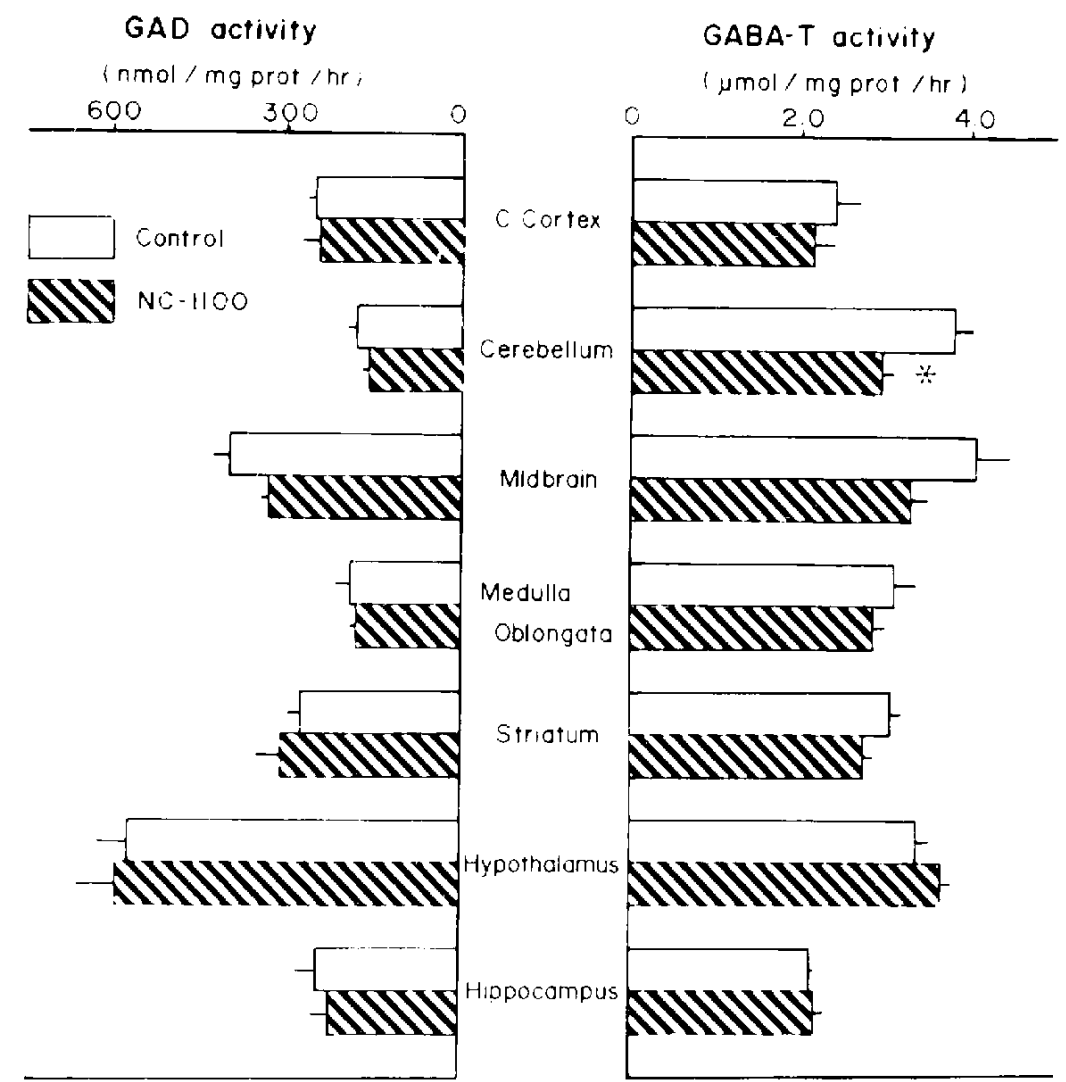

Fig. 3. Effect of continuous oral administration of NC-1100 on glutamic acid decarboxylase (GAD) and GABA-transaminase (GABA-T) activities in the brain of SHR-SP. One hundred $\mathrm{mg} / \mathrm{kg}$ of $\mathrm{NC}-1100$ was administered orally once a day for 7 days. Each value represents the mean \pm S.E.M. obtained from 4 separate experiments. $* P<0.05$, compared with the control value. 


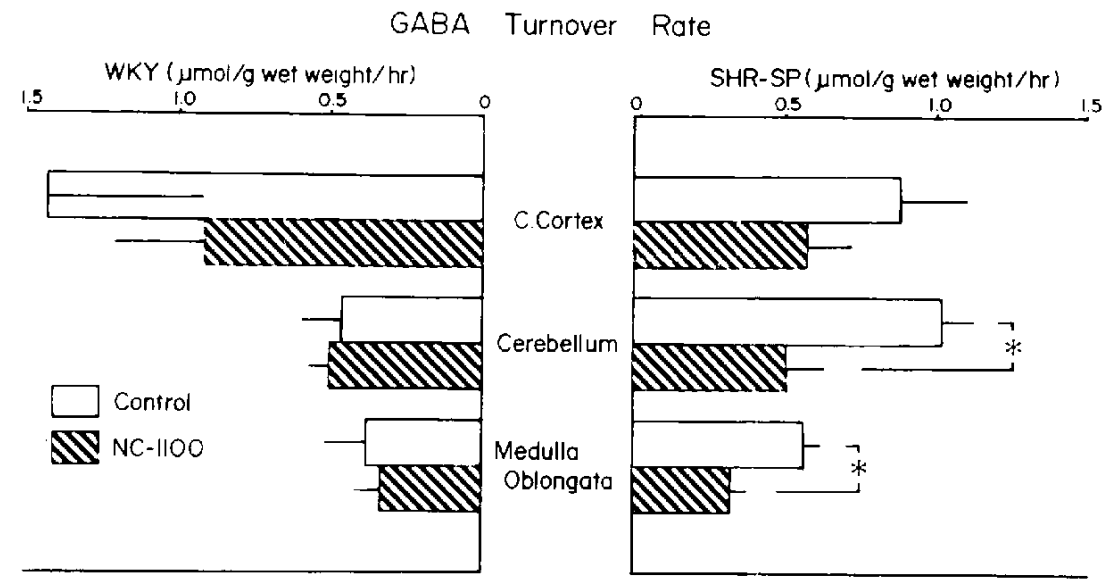

Fig. 4. Effect of continuous oral administration of NC-1100 on GABA tupnover rate in brains of WKY and SHR-SP. Rats recieved $100 \mathrm{mg} / \mathrm{kg}$ of NC-1100 once daily for 7 days. Each value represents the mean \pm S.E.M. obtained from 4 separate experiments. ${ }^{*} P<0.05$, compared with each control value.

of GABA was detected, however, in the cerebral cortex, cerebellum and medulla oblongata of NC-1100 treated WKY.

Effect of continuous oral administration of NC-1100 on uptake and release of GABA: Since continuous oral administration of NC1100 to SHR-SP was found to induce an increment of GABA in the cerebellum and medulla oblongata inspite of the decrease of turnover rate of GABA in these brain areas, the uptake and release of $\left[{ }^{3} \mathrm{H}\right] \mathrm{GABA}$ were examined using slices of the cerebellum, medulla oblongata and cerebral cortex of SHR-SP.

The uptake of [3H] GABA to these slices was found to be not affected by the administration of $\mathrm{NC}-1100(100 \mathrm{mg} / \mathrm{kg}$, p.o., 7 days). On the other hand, NC-1100 administration caused a significant increase in the half value of the component $\left(t_{1 / 2}\right)$ in the slow phase of $\left[{ }^{3} \mathrm{H}\right] \mathrm{GABA}$ release from slices of the cerebellum and medulla oblongata of SHR-SP (Table 2). In contrast, the spontaneous release of $\left[{ }^{3} \mathrm{H}\right] \mathrm{GABA}$ (Table 2 ) as well as metabolic turnover rate of GABA (Fig. 4) in the cerebral cortex of SHR-SP showed no significant alteration following the continuous administration of NC-1100. In the cerebellum and medulla oblongata, it was also found that the continuous administration of $\mathrm{NC}$ 1100 has no significant effect not only on the release of $\left[{ }^{3} \mathrm{H}\right] \mathrm{GABA}$ in the rapid phase but also that observed under high potassium (30 $\mathrm{mM}$ )-depolarization in all animals examined (Table 2).

Alteration of $\left[{ }^{3} \mathrm{H}\right]$ muscimol binding to cerebral synaptic membrane of SHR-SP following continuous administration of $\mathrm{NC}$ 1100: Continuous administration of NC-1100 (100 mg/kg, p.o., 7 days) did not alter the high affinity and specific binding of $\left[{ }^{3} \mathrm{H}\right]$ muscimol to synaptic membranes obtained from the cerebellum, medulla oblongata and other brain regions.

\section{Discussion}

$\mathrm{NC}-1100$ is a drug that was developed for the treatment of cerebrovascular diseases and known to have a potency to increase the blood flow of vertebral and upper jaw arteries in a dose-dependent manner following intravenous and intraarterial administrations (10).

One of the interesting findings obtained in this study is that continuous oral administration of NC-1100 induces the increase of GABA in the cerebellum and medulla oblongata of SHR-SP.

GABA, glutamic acid and aspartic acid are known to have important functions not only as a neurotransmitter (11-13) but also as metabolic intermediates associated with the TCA cycle in the mammalian CNS. In the brain of SHR-SP, it was found that GABA contents in the cerebellum, hypothalamus 
and hippocampus showed a significant decrease as compared with those of WKY (Table 1). Hambley et al. (14) also reported that the GABA content in the hypothalamus of SHR showed a significant decrease at 75 and 120 days after birth. Furthermore. Sasaki et al. (15) reported that intracerebroventricular (i.c.v.) injection of GABA reduced the sympathetic nerve activity, arterial blood pressure, and heart rate in a dose-dependent manner in normotensive WKY. In the SHR, intraventricularly injected GABA also reduced sympathetic and cardiovascular activities, but the magnitude of these effects was found to be significantly larger than those found in WKY (15). Considering these facts, it is probable that the decrease of GABA content in the hypothalamus of SHR or SHR-SP may be indicative of the dysfunction of the hypothalamic GABA system in spontaneously hypertensive rats. On the other hand, there have been no reports on the decrease of GABA in the cerebellum and hyppocampus of SHR. We have found that the decrease of GABA in the cerebellum of SHR-SP is due to the increase of GABA turnover, because the turnover rate of the cerebellar GABA in SHR$S P$ showed a significant increase $(P<0.05)$ as compared with that of WKY. Similar increase of GABA turnover was also observed in the medulla oblongata of SHR-SP

In the SHR-SP, the continuous oral administration of NC-1100 induced the decrease of the activity of GABA-T, the GABA degrading enzyme, without affecting that of $G A D$, the GABA synthesizing enzyme, in the cerebellum and medulla oblongata of SHR-SP. In addition, it was observed that the turnover rate of GABA in the cerebellum and medulla oblongata of SHR-SP was decreased by the NC-1100 treatment. On the other hand, the continuous administration of NC-1100 induced a significant reduction of the spontaneous release of preloaded $\left[{ }^{3} \mathrm{H}\right] \mathrm{GABA}$ from slices of the cerebellum and medulla oblongata of SHR-SP without affecting the uptake. Considering the results obtained in this study, it seems possible that the elevation of GABA contents in the cerebellum and medulla oblongata of SHR-SP following NC1100 administration may be due to the decrease of GABA-T activity as well as the retardation of spontaneous release of GABA. Since continuous administration of NC-1100 induced significant increase of GABA in the cerebellum and medulla oblongata of SHR$\mathrm{SP}$. possible alteration in the high affinity binding of $\left[{ }^{3} \mathrm{H}\right]$ muscimol to $\mathrm{GABA}_{\Lambda}$ receptors was also examined. The treatment with NC-1100, however, did not induce any significant changes in the binding of $\left[{ }^{3} \mathrm{H}\right]$ muscimol not only in the synaptic membrane preparations obtained from the cerebellum and medulla oblongata but also those from other brain areas. The lack of effect of NC1100 treatment on GABA receptor binding. in addition to no alteration of a high potassium-evoked release of GABA under the same experimental conditions, suggests that the observed increase of GABA contents in the cerebellum and medulla oblongata of SHRSP may be due to the inhibition of metabolic degradation of GABA rather the accentuation of synthesis and/or metabolic degradation of GABA. In fact, this view seems supported by the observed facts that NC-1100 treatment inhibits the activity of GABA-T, a GABA degrading enzyme, but not that of GAD, a GABA synthesizing enzyme.

Besides the increase of GABA in the cerebellum and medulla oblongata of SHR$\mathrm{SP}$, the decrease of aspartic acid was noted in the same brain areas following continuous NC-1100 administration. The cause of this decrease in aspartic acid content is not clear at present, but it seems likely to be related to the observed decreases in GABA-T activity and GABA turnover, since aspartic acid is synthesized by means of the TCA cycle to which the so-called GABA shunt is metabolically interconnected (16). The clarification of this point, however, remains for future studies.

In conclusion, NC-1100 induces the increase of GABA in the cerebellum and medulla oblongata through the inhibition of its metabolic turnover if it is administered to SHR$S P$. These results suggest that the possible therapeutic efficacy of this drug on various neurological disorders associated with cerebral hemorrhage and/or arteriosclerosis may involve functional and/or metabolic alterations in GABA containing neurons in these brain regions. 


\section{References}

1 Olesen, J. and Paulson, O.B.: The effect of intraarterial papaverine on regional cerebral blood flow in patients with stroke or intracranial tumor. Stroke 2, 148-155 (1971)

2 Kuriyama, K: Taurine as a neuromodulator. Fed. Proc. 39, 2680-2684 (1980)

3 Glowinski, J. and Iversen, L. L.: Regional studies of catecholamines in the rat brain 1. The disposition of $\left[{ }^{3} \mathrm{H}\right]$ norepinephrine, $\left[{ }^{3} \mathrm{H}\right]$ dopa in various regions of the brain. $J$. Neurochem. 13, 655-669 (1966)

4 Ida, S. and Kuriyama, K.: Simultaneous determination of cysteine sulfinic acid and cysteic acid in the rat brain by high performance liquid chromatography. Anal. Biochem. 130, 95-101 (1983)

5 Kimura, $H$. and Kuriyama, K.: A new microassay method for L-glutamic acid decarboxylase (GAD) activity. Japan. J. Pharmacol. 25, 189-195 (1975)

6 Löscher, W.: Correlation between alterations in brain GABA metabolism and seizure excitability following administration of GABA aminotransferase inhibitors and valproic acid- a reevaluation. Neurochem. int. 3, 397-404 (1981)

7 Kuriyama, K., Muramatsu, M., Nakagawa, K. and Kakita, K.: Modulating role of taurine on release of neurotransmitters and calcium transport in excitable tissue. In Taurine and Neurological Disorders, Edited by Barbeau, A. and Huxtable. R.J., p. 201-216, Raven Press, New York (1978)

8 Ito, Y. and Kuriyama, K.: Some properties of solubilized GABA receptor. Brain Res. 236, 351-363 (1982)

9 Lowry, O.H., Rosebrough, N.J., Farr, A.L. and
Randal!, R.J.: Protein measurement with the Folin phenol reagent. J. Biol. Chem. 193, 265275 (1951)

10 Kushiku, K., Araki, Y., Mikagi, T., Ohba, M., Katsuragi, T. and Furukawa, T.: Cardiovascular effects of NC-1100 and possible modes of action. Folia Pharmacol. Japon. 87, 111P-112P (1986) (in Japanese)

11 Iversen, L.L., Mitchel!, J.F. and Srinivasan, V.: The release of $\gamma$-aminobutyric acid during inhibition in the cat visual cortex. J. Physiol. (Lond.) 212, 519-534 (1971)

12 Mulder, A.H. and Snyder, S.H.: Potassiuminduced release of amino acids from cerebral cortex and spinal cord slices of the rat. Brain Res. 76, 297-308 (1974)

13 Hamberger, A.C., Chiang, G.H., Nylen, E.S., Scheff, S.W. and Cotman, C.W.: Glutamate as a CNS transmitter I. Evaluation of glucose and glutamate as precursors for the synthesis of preferentially released glutamate. Brain Res. 168, 513-530 (1979)

14 Hambley, G.W., Johnston, G.A.R. and Shaw, J.: Alteration in a hypothalamic GABA system in the spontaneously hypertensive rat. Neurochem. Int. 6, 813-821 (1984)

15 Sasaki, S., Lee, L.C., Nakamura, Y., lyota, I., Fukuyama, M., Inoue, A., Takeda, K., Yoshimura, M. Nakagawa, M. and ljichi, H.: Hypotension and hypothalamic depression produced by intracerebroventricular injections of GABA in spontaneously hypertensive rats. Japan. Cir. Res. 50, 1140-1148 (1986)

16 McGreen, P.L. and McGreen, E.G.: Amino acid neuratransmitters. In Brain Neurotransmitters, Third Edition, Edited by Siegal, G.T. et al., p. 233253, Little, Brown and Company, Boston (1981) 